\title{
Predictors of postoperative infectious complica- tions in liver transplant recipients: experience of 185 consecutive cases
}

Seungjin Lim ${ }^{1,2}$, Eun Jung Kim ${ }^{1,2}$, Tae Beom Lee ${ }^{3}$, Byung Hyun Choi ${ }^{3}$, Young Mok Park ${ }^{3}$, Kwangho Yang ${ }^{3}$, Je Ho Ryu ${ }^{3}$, Chong Woo Chu ${ }^{3}$, and Su Jin Lee ${ }^{1,2}$

${ }^{1}$ Department of Internal Medicine, Pusan National University School of Medicine, Yangsan; ${ }^{2}$ Research Institute for Convergence of Biomedical Science and Technology, ${ }^{3}$ Department of Surgery, Pusan National University Yangsan Hospital, Yangsan, Korea
Received: July 5, 2017

Revised : September 5, 2017

Accepted: September 28, 2017

\section{Correspondence to}

\section{Su Jin Lee, M.D.}

Department of Internal Medicine, Pusan National University Yangsan Hospital, 20 Geumo-ro, Mulgeum-eup, Yangsan 50612, Korea

Tel: $+82-55-360-1626$

Fax: +82-55-360-2150

E-mail: beauty192@hanmail.net
Background/Aims: Infections following liver transplant (LT) remain a major cause of mortality. This study was conducted to evaluate risk factors for infection and to review clinical characteristics.

Methods: Medical records of patients who underwent LT from 2010 to 2014 were retrospectively analyzed. Binary logistic regression analysis was used to investigate risk factors of infection. Kaplan-Meier analysis was used to predict prognosis of infected and non-infected groups.

Results: Of 185 recipients, 89 patients experienced infectious complications. The median follow-up period was 911 days (range, 9 to 2,031$)$. The infected group had higher 1-year mortality $(\mathrm{n}=22[24.7 \%]$ vs. $\mathrm{n}=8,[8.3 \%], \mathrm{p}=0.002)$, and longer postoperative admission days (mean: $53.7 \pm 35.8$ days vs. $28.3 \pm 13.0$ days, $\mathrm{p}<0.001$ ), compared to the non-infected group. High preoperative Model for End-Stage Liver Disease (MELD) score (odds ratio [OR], 1.057; 95\% confidence interval [CI], 1.010 to $1.105 ; p=0.016)$, deceased-donor type (OR, 5.475; 95\% CI, 2.442 to 12.279 ; $p<0.001$ ), and acute rejection (OR, 3.042; 95\% CI, 1.241 to $7.454 ; p=0.015$ ) were independent risk factors associated with infection. Intra-abdominal infection ( $\mathrm{n}=$ $35,20.8 \%$ ) was the major infectious complication. Among identified bacteria, Enterococcus species (28.4\%) were major pathogens, followed by Escherichia coli and Klebsiella species.

Conclusions: High preoperative MELD score, deceased-donor type, and acute rejection were risk factors associated with infection. To prevent infections following surgery, it is important to determine the appropriate time of operation before the recipient has a high MELD score.

Keywords: Liver transplantation; Infection; Risk factors

\section{INTRODUCTION}

Despite continued improvement in clinical management of liver transplant (LT) recipients, infections continue to be one of the leading causes of morbidity and mortality [1]. LT recipients are more likely to develop bacterial infections than other organ transplant recipients due to the complexity of the surgical procedure which includes penetration of the hepatobiliary system [2]. Adverse outcomes within the first year after liver transplantation are primarily caused by allograft-related complications and infections [3]. Diagnosis of infection 
in these patients is difficult because the signs and symptoms are usually mild or often absent [2]. Nevertheless, even mild infections in immunocompromised patients can have catastrophic effects [4]. During the initial period following LT, there is an increased incidence of central line-associated bloodstream infection (CLABSI) or ventilator associated pneumonia (VAP) [4]. In addition, the emergence of multi-drug resistant organisms worldwide in many transplant centers is a major problem requiring aggressive management [4]. The aim of this study therefore was to determine factors associated with infections after liver transplantation, and to review the clinical and microbiological characteristics of those infections.

\section{METHODS}

\section{Study design and setting}

Medical records of 185 patients who underwent liver transplantation from May 2010 to December 2014 at Pusan National University Yangsan Hospital, Yangsan, Korea, were retrospectively reviewed. The types of infectious complications and causes of deaths were reviewed during a follow-up period until December 2015.

Demographic and baseline characteristics, laboratory data, outcomes, and infectious complications were collected. Model for End-Stage Liver Disease (MELD) score was calculated using the following formula: $3.78 \times \log _{\mathrm{e}}$ (bilirubin, $\mathrm{mg} / \mathrm{dL}$ ) $+11.2 \times \log _{\mathrm{e}}$ (international normalized ratio $[\mathrm{INR}])+9.57 \times \log _{\mathrm{e}}($ creatinine, $\mathrm{mg} / \mathrm{dL})+6.43$ [3]. Creatinine level was converted to $4 \mathrm{mg} / \mathrm{dL}$ the day after initiation and at 3 days after the last use of renal replacement therapy if the procedure was done more than once [3]. This study was approved by the Institutional Review Board of Pusan National University Yangsan Hospital (Approval No. 30-2015-011). Informed consent was waived by the board.

\section{Standard post-transplant management Immunosuppression regimen}

Basiliximab at 1 day pre-LT and 4 days post-LT was used for induction of immunosuppression. Tacrolimus or cyclosporine with steroid was used as the maintenance immunosuppressant regimen in our hospital. The dose of tacrolimus was adjusted to maintain a blood level of
13 to $15 \mu \mathrm{g} / \mathrm{L}$ for 2 weeks postoperatively and 10 to $13 \mu \mathrm{g} / \mathrm{L}$ thereafter. Blood levels of cyclosporine were maintained at $200 \mu \mathrm{g} / \mathrm{L}$ for 2 weeks after surgery and then maintained at 150 to $200 \mu \mathrm{g} / \mathrm{L}$. Additionally, methylprednisolone $(3 \mathrm{mg} / \mathrm{kg}$ ) was administered intravenously on the first day after LT. Its dose was gradually tapered until the 11th day after LT.

\section{Antimicrobial prophylaxis}

The standard surgical antibiotic prophylaxis regimen at our institution varied according to individual patient's MELD score. Piperacillin/tazobactam and ciprofloxacin were given as prophylactic antibiotics to patients with MELD scores of less than 25, while vancomycin and meropenem were administered to patients with MELD score of 25 or higher. All patients received prophylactic antibiotics for 7 days unless there were other signs of infection. For antifungal prophylaxis, amphotericin B or liposomal amphotericin B were administered to recipients for 7 days after the surgery followed by administration of itraconazole for 2 weeks. Antiviral prophylaxis including ganciclovir was not administered except for seronegative recipients who received an allograft from cytomegalovirus (CMV)-seropositive donor [5]. For recipients with results positive for CMV by quantitative polymerase chain reaction (CMV-qPCR, VERI/MDx System CMV Assay, Beckman Coulter, Danvers, MA, USA) in the follow-up period, pre-emptive treatment with ganciclovir or valganciclovir was used depending on renal function. All patients were monitored by CMV qPCR weekly for the first 2 months and twice monthly up to 6 months after the transplant. All patients were administered $80 / 400 \mathrm{mg}$ trimethoprim-sulfamethoxazole once a day for 3 months post-transplant as prophylaxis for Pneumocystis jirovecii pneumonia.

\section{Definitions for infectious complications}

Infectious complications were defined and categorized according to established consensus recommendations, or Centers for Disease Control and Prevention criteria [6]. Based on clinical and microbiological documentation from patients' medical records, all infectious syndromes were categorized into one of the following: respiratory, bloodstream (due to central line or other causes), genitourinary, intra-abdominal (including infection of hepatobiliary system), surgical site, and oth- 
Table 1. Demographic features and clinical characteristics of patients

\begin{tabular}{|c|c|c|c|c|}
\hline Parameter & $\begin{array}{l}\text { All patients } \\
\qquad(\mathrm{n}=185)\end{array}$ & $\begin{array}{l}\text { Non-infected group } \\
\qquad(\mathrm{n}=96)\end{array}$ & $\begin{array}{l}\text { Infected group } \\
\qquad(\mathrm{n}=89)\end{array}$ & $p$ value \\
\hline Age, yr & $53(10-69)$ & $52(14-67)$ & $55(10-69)$ & 0.107 \\
\hline Sex, male/female & $131(70.8) / 54(29.2)$ & $73(75 \cdot 3) / 24(24 \cdot 7)$ & $58(65.9) / 30(34.1)$ & 0.196 \\
\hline \multicolumn{5}{|l|}{ Etiology of liver disease } \\
\hline HBV & $110(59 \cdot 5)$ & $70(72.2)$ & $40(45 \cdot 5)$ & $<0.001$ \\
\hline $\mathrm{HCV}$ & $22(11.9)$ & $9(9 \cdot 3)$ & $13(14.8)$ & 0.177 \\
\hline Alcohol & $38(20.5)$ & $20(20.6)$ & $18(20.5)$ & 0.562 \\
\hline Autoimmune & $5(2.7)$ & $1(0.5)$ & $4(2.2)$ & 0.155 \\
\hline Idiopathic & $6(3.2)$ & $2(2.1)$ & $4(4 \cdot 5)$ & 0.426 \\
\hline $\mathrm{HCC}$ & $80(43.2)$ & $51(63.7)$ & $29(36.3)$ & 0.008 \\
\hline MELD score & $17.57 \pm 11.53$ & $13.87 \pm 9.79$ & $21.6 \pm 11.97$ & $<0.001$ \\
\hline Child Pugh score & $4.58 \pm 3.72$ & $4.01 \pm 3.34$ & $5.22 \pm 4.02$ & 0.029 \\
\hline \multicolumn{5}{|l|}{ Type of donor } \\
\hline Deceased donor & $76(41.1)$ & $21(21.6)$ & $55(62.5)$ & $<0.001$ \\
\hline Living donor & $106(58.9)$ & $76(78.4)$ & $33(37 \cdot 5)$ & \\
\hline \multicolumn{5}{|c|}{ Preoperative laboratory finding } \\
\hline ALT, IU/L & $35.0(4-3,280)$ & $34.0(4.0-3,280)$ & $36.0(4-1,980)$ & 0.898 \\
\hline ALP, IU/L & $220.0(34-1,761)$ & $252(133-579)$ & $239.0(34-1,761)$ & 0.095 \\
\hline Creatinine, $\mathrm{mg} / \mathrm{dL}$ & $1.0 \pm 0.7$ & $0.91 \pm 0.5$ & $1.14 \pm 0.75$ & 0.021 \\
\hline PT-INR & $1.80 \pm 0.70$ & $1.69 \pm 0.79$ & $1.92 \pm 0.66$ & 0.029 \\
\hline Platelet, $10^{3} / \mu \mathrm{L}$ & $79.0 \pm 49 \cdot 5$ & $81.72 \pm 43.23$ & $76.06 \pm 55.63$ & 0.439 \\
\hline $\mathrm{CRP}, \mathrm{mg} / \mathrm{dL}$ & $0.63(0.06-18.87)$ & $0.61(0.08-2.54)$ & $1.28(0.10-18.87)$ & $<0.001$ \\
\hline $\mathrm{BNP}, \mathrm{pg} / \mathrm{mL}$ & $70.6(7-3,728)$ & $49(7-2,094)$ & $102(12-3,728)$ & 0.011 \\
\hline $\begin{array}{l}\text { Period from surgery to } \\
\text { discharge, day }\end{array}$ & $29.0(3-188)$ & $26.5(8-116)$ & $45 \cdot 0(3-188)$ & $<0.001$ \\
\hline Duration of ICU & $12.0(3-72)$ & $5(3-54)$ & $18.0(3-72)$ & $<0.001$ \\
\hline Duration of ventilator care & $4.0(0-57)$ & $2(0-34)$ & $9(0-57)$ & $<0.001$ \\
\hline ECHMO & $8(4 \cdot 3)$ & $1(1.0)$ & $7(8.0)$ & 0.027 \\
\hline Acute rejection & $36(19 \cdot 5)$ & $13(13 \cdot 4)$ & $23(26.1)$ & 0.040 \\
\hline \multicolumn{5}{|c|}{ Laboratory variable on postoperative 3 rd day } \\
\hline ALT, IU/L & $177(25-3,728)$ & $133(56-700)$ & $198.0(25-3,728)$ & 0.039 \\
\hline ALP, IU/L & $131.0(55-3,805)$ & $112.0(55-3,805)$ & $193(64-2,422)$ & 0.033 \\
\hline Creatinine, mg/dL & $1.05 \pm 0.44$ & $0.99 \pm 0.41$ & $1.11 \pm 0.46$ & 0.085 \\
\hline PT-INR & $1.40 \pm 0.61$ & $1.43 \pm 0.82$ & $1.37 \pm 0.23$ & 0.504 \\
\hline Platelet, $10^{3} / \mu \mathrm{L}$ & $46.01 \pm 20.92$ & $47.00 \pm 16.40$ & $44.95 \pm 24.95$ & 0.508 \\
\hline CRP, mg/dL & $2.75(0.12-29.70)$ & $2.36(0.50-7.76)$ & $3.29(0.12-29.70)$ & 0.385 \\
\hline Follow-up period, day & $911(2-2,031)$ & $948.5(2-1,917)$ & $873(2-2,031)$ & 0.213 \\
\hline 30-Day mortality & $11(6.0)$ & $1(3.1)$ & $10(9.2)$ & 0.073 \\
\hline 1-Year mortality & $30(16.2)$ & $8(8.3)$ & $22(24.7)$ & 0.002 \\
\hline
\end{tabular}

Values are presented as median (range), number (\%), or mean \pm SD.

HBV, hepatitis B virus; HCV, hepatitis C virus; HCC, hepatocellular carcinoma; MELD, Model for End-Stage Liver Disease; ALT, alanine aminotransferase; ALP, alkaline phosphatase; PT-INR, prothrombin time-international normalized ratio; CRP, C-reactive protein; BNP, brain natriuretic peptide; ICU, intensive care unit; ECHMO, extracorporeal membrane oxygenation. 
ers. Absence or presence of fungal infection and viral infection were also reviewed. CMV disease was defined following the guidelines proposed by Ljungman et al. [7]. It included either viral syndrome or end-organ disease. Invasive fungal infection was defined using the criteria proposed by the European Organization on Research and Treatment in Cancer and the Mycoses Study Group [8]. In cases where no microbiological etiology was established, clinical and radiological documentation were used to categorize infections.

\section{Outcomes}

We assessed the incidence of postoperative infectious complications, 30-day mortality and 1-year mortality following LT. Patient deaths after one year and during the follow-up period was further investigated.

\section{Statistical analysis}

Student $t$ test was used to analyze continuous variables and chi-square test was used to analyze categorical variables. Variables with $p<0.05$ in the univariate analysis were included in the multivariate analysis. Multivariate analysis used binary logistic regression tests. The Kaplan-Meier method with log-rank test was used to analyze the survival of patients with infectious complication and those without infectious complications. Statistical analysis was performed using SPSS version 21.0 for Windows (IBM Co., Armonk, NY, USA). Statistical significance was considered achieved at $p$ values less than 0.05 .

\section{RESULTS}

\section{Demographic features of the infected and non-in- fected patient groups}

During the study period, there were 188 liver transplantations in 185 patients. Demographic, clinical, and laboratory characteristics of these 185 patients in the preoperative and postoperative periods are summarized in Table 1. The median age of patients was 53 years (range, 10 to 69 ). There were 131 male patients (70.8\%). Age and gender were not significantly different between infected and non-infected groups. Of these 185 patients, 110 patients (59.5\%) had cirrhosis or chronic hepatitis B and 80 patients $(43.2 \%)$ had hepatocellular carcinoma (HCC). A total of 76 patients received organs from deceased-do-

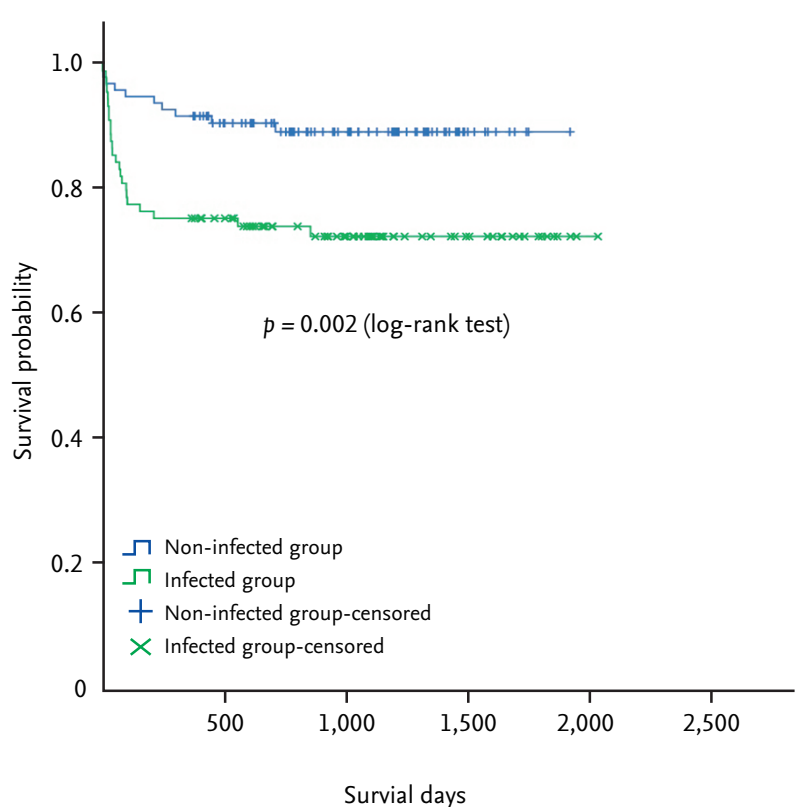

Figure 1. Kaplan-Meier survival curves between infected and non-infected groups of liver transplant recipients.

nors (41.1\%). The median follow-up duration of all patients was 911 days (range, 2 to 2,031). The 1-year all-cause mortality was $16.2 \%$. A total of 89 patients experienced infections (48.1\%; 173 infectious episodes) during the follow-up period. There were significant differences in the incidence of hepatitis B virus (HBV) infection and HCC between the infected and non-infected groups. HBV infection and HCC were more frequently observed in the non-infected group (HBV: $72.2 \%$ vs. $45.5 \%$ in infected, $p$ $<0.001$; HCC: $65.7 \%$ vs. $36.3 \%$ in infected, $p=0.008$ ). On the other hand, hepatitis $\mathrm{C}$ virus infection, alcoholic liver diseases, and autoimmune liver diseases did not show a significant difference in prevalence rate between the two groups. The number of patients who received transplants from deceased-donors in the infected group was higher than that in the non-infected group $(n=55,62.5 \%$ in the infected group vs. $\mathrm{n}=21,21.6 \%$ in the non-infected group, $p<0.001$ ).

MELD scores and Child-Pugh scores were both higher in the infected compared to the non-infected groups $(21.60 \pm 11.97$ in infected vs. $13.87 \pm 9.79$ in the non-infected, $p<0.001 ; 5.22 \pm 4.02$ in infected vs. $4.01 \pm 3.34$ in non-infected, $p=0.029$, respectively). There were significant differences in creatinine level $(0.91 \pm 0.05 \mathrm{mg} / \mathrm{dL}$ in non-infected vs. $1.14 \pm 0.75 \mathrm{mg} / \mathrm{dL}$ in infected, $p=0.021)$ and prothrombin time-international normalized ratio (PT- 
Table 2. Multivariate analysis of infectious complications-related factors

\begin{tabular}{lccc}
\hline \multirow{2}{*}{ Variable } & & Non-infected vs. infected & p value \\
\cline { 2 - 4 } HBV & Odds ratio & $95 \%$ CI & 0.257 \\
HCC & 0.656 & $0.316-1.359$ & 0.778 \\
MELD score & 1.134 & $0.473-2.721$ & 0.016 \\
Child Pugh score & 1.057 & $1.010-1.105$ & 0.833 \\
Deceased-donor & 1.011 & $0.912-1.121$ & $<0.001$ \\
Creatinine, mg/dL & 5.475 & $2.442-12.279$ & 0.784 \\
PT-INR & 1.093 & $0.579-2.065$ & 0.063 \\
CRP, mg/dL & 0.516 & $0.257-1.036$ & 0.705 \\
BNP, pg/mL & 1.034 & $0.869-1.231$ & 0.451 \\
Acute rejection & 1.000 & $0.999-1.001$ & 0.015 \\
ALT (3 days after LT), IU/L ${ }^{a}$ & 3.042 & $1.241-7.454$ & 0.384 \\
ALP (3 days after LT), IU/L ${ }^{\mathrm{a}}$ & 1.000 & $0.999-1.001$ & 0.824 \\
\hline
\end{tabular}

CI, confidence interval; HBV, hepatitis B virus; HCC, hepatocellular carcinoma; MELD, Model for End-Stage Liver Disease; PT-INR, prothrombin time international normalized ratio; CRP, C-reactive protein; BNP, brain natriuretic peptide; ALT, alanine aminotransferase; LT, liver transplant; ALP, alkaline phosphatase.

${ }^{\mathrm{a}}$ Laboratory variables were taken at 3 days after LT.

INR) (1.69 \pm 0.79 non-infected vs. $1.92 \pm 0.66$ infected, $p$ $=0.029)$. These two laboratory variables are involved in the MELD score. C-reactive protein level (median, 0.61 $\mathrm{mg} / \mathrm{dL}$ [range, 0.08 to 2.54 ] in non-infected vs. median, $1.28 \mathrm{mg} / \mathrm{dL}$ [range, 0.10 to 18.87 ] in infected, $p<0.001$ ) and brain natriuretic peptide level $(49 \mathrm{pg} / \mathrm{mL}$ [range, 7 to 2,094] in non-infected vs. $102 \mathrm{pg} / \mathrm{mL}$ (range, 12 to 3,728 ] in infected, $p=0.011$ ) also showed significant differences.

In terms of postoperative clinical features, the group with infectious complications had a higher acute rejection rate $(n=23,26.1 \%$ vs. $n=13,13.4 \%$ in non-infected, $p=$ 0.040 ), higher 1-year mortality ( $24.7 \%$ vs. $8.3 \%$ in non-infected, $p=0.002$ ), longer post-operative admission days $(53.7 \pm 35.8$ days vs. $28.3 \pm 13.0$ days in non-infected, $p<$ 0.001), and longer intensive care unit (ICU) stays (22.1 \pm 14.3 days vs. $10.0 \pm 7.0$ days in non-infected, $p<0.001$ ). During ICU care, longer ventilator duration was needed for the infected group compared to the non-infected group (13.0 \pm 12.3 days vs. $4.0 \pm 4.9$ days in non-infected, $p$ $<0.001$ ). In addition, extracorporeal membrane oxygenation was more frequently applied in the infected group ( $\mathrm{n}=7,8 \%$ in the infected group vs. $\mathrm{n}=1,1.0 \%$ in non-infected group, $p=0.027$ ). In Kaplan-Meier analysis, there was a significant difference in survival rate between the two groups $(p=0.002)$ (Fig. 1 ).

\section{Risk factors for infectious morbidity}

Based on these results, multivariate analysis was performed to identify independent risk factors for infectious complications. Results are summarized in Table 2. As preoperative MELD score increased, the risk of infection also increased (odds ratio [OR], 1.057; 95\% confidence interval [CI], 1.010 to $1.105 ; p=0.016)$. Presence of acute rejection (OR, 3.042; 95\% CI, 1.241 to $7.454 ; p=$ 0.015) and deceased donor type (OR, 5.475; 95\% CI, 2.442 to $12.279 ; p<0.001$ ) were also found to be risk factors for infectious complications in this study. However, no single laboratory result was found to be an independent predictor of infection.

\section{Characteristics of infectious complications in liver transplant recipients}

A total of 89 patients experienced 168 infectious episodes during the follow-up period (48.2\%). The number of episodes of infectious complications and the number of pathogens are summarized in Tables 3 and 4 . Bacteria were the most commonly identified causative agents in infectious complications ( $\mathrm{n}=180,90.9 \%$ of total identified organisms), followed by fungi $(\mathrm{n}=15,7.5 \%)$ and viruses $(n=4,2.0 \%)$. Intra-abdominal infection $(n=$ $35,20.8 \%$ of total episodes of infection, $0.038 / 1,000$ pa- 
tient-month) was the most common type of infectious complication, followed by urinary tract infection $(n=30$,
$17.8 \%, 0.032 / 1,000$ patient-month), pneumonia $(\mathrm{n}=29$, $17.2 \%, 0.031 / 1,000$ patient-month), and CLABSI $(\mathrm{n}=24$,

Table 3. Infectious complication after liver transplantation

\begin{tabular}{|c|c|c|c|c|c|}
\hline \multirow{2}{*}{ Types of infection } & \multicolumn{3}{|c|}{ No. of episodes of infection } & \multirow{2}{*}{$\begin{array}{c}\text { Total } \\
(\mathrm{n}=168)\end{array}$} & \multirow{2}{*}{$\begin{array}{c}\text { Incidence } \\
\text { (1,000 patient-month) }\end{array}$} \\
\hline & $<1$ Month & 1-3 Months & $>3$ Months & & \\
\hline Intra-abdominal infection & 22 & 9 & 4 & $35(20.8)$ & 0.038 \\
\hline CLABSI & 18 & 5 & 1 & $24(14.2)$ & 0.026 \\
\hline BSI except CLABSI & 21 & 8 & 3 & $32(19.0)$ & 0.034 \\
\hline Pneumonia & 25 & 2 & 2 & $29(17.2)$ & 0.031 \\
\hline UTI & 20 & 8 & 2 & $30(17.8)$ & 0.032 \\
\hline Wound infection & 9 & o & o & $9(5 \cdot 3)$ & 0.010 \\
\hline Others & 5 & 2 & 2 & $9(5 \cdot 3)$ & 0.010 \\
\hline Subtotal & $120(71.4)$ & $34(20.2)$ & $14(8.3)$ & & 0.181 \\
\hline
\end{tabular}

Values are presented as number (\%).

CLABSI, central line-associated bloodstream infection; BSI, bloodstream infection; UTI, urinary tract infection.

Table 4. Infectious organisms after liver transplantation during different time periods

\begin{tabular}{|c|c|c|c|c|}
\hline Organism & $<1$ Month & 1-3 Months & $>3$ Months & Total \\
\hline Total no. (\%) & $154(70.6)$ & $44(20.1)$ & $20(9.1)$ & 218 \\
\hline Gram-positive bacteria & & & & 86 \\
\hline Enterococcus species & 41 & 7 & 3 & 51 \\
\hline Coagulase-negative staphylococci & 13 & 2 & 2 & 17 \\
\hline Staphylococcus aureus & 12 & 1 & 1 & 14 \\
\hline Clostridium difficile & 1 & 1 & 0 & 2 \\
\hline Other Gram-positive bacteria & 1 & 1 & 0 & 2 \\
\hline Gram-negative bacteria & & & & 91 \\
\hline Escherichia coli & 12 & 5 & 2 & 19 \\
\hline Klebsiella species & 17 & 7 & 2 & 26 \\
\hline Enterobacter species & 2 & o & o & 2 \\
\hline Pseudomonas aeruginosa & 7 & 2 & 0 & 9 \\
\hline Acinetobacter baumannii & 14 & 2 & o & 16 \\
\hline Stenotrophomonas maltophilia & 13 & 4 & o & 17 \\
\hline Other Gram-negative organism & 1 & 1 & o & 2 \\
\hline Mycobacterium tuberculosis & 1 & 1 & ० & 2 \\
\hline Fungi & & & & 15 \\
\hline Candida species & 7 & 4 & 2 & 13 \\
\hline Aspergillus species & 1 & o & 1 & 2 \\
\hline Viruses & & & & 4 \\
\hline Cytomegalovirus & 1 & 1 & o & 2 \\
\hline Herpes simplex virus & 0 & o & 1 & 1 \\
\hline Ebstein-Barr virus & $\mathrm{O}$ & $\mathrm{O}$ & 1 & 1 \\
\hline Unknown & 10 & 5 & 5 & 20 \\
\hline
\end{tabular}


$14.2 \%, 0.026 / 1,000$ patient-month). A small number of infections involving other organs were reported, including central nervous system infection and musculoskeletal infection. Of the total infectious complications, $71.4 \%$ occurred within 1 month of the operation. Infections acquired 3 months after the operation accounted for $8.3 \%$ of the total.

Among microbiologically-documented intra-abdominal infections, Enterococcus species were found most frequently $(\mathrm{n}=17,49.3 \%)$, followed by Escherichia coli $(\mathrm{n}=4)$ and Klebsiella species $(\mathrm{n}=4)$. Among urinary tract infections in 30 patients, Enterococcus species were found in 10 of them. Klebsiella species and E. coli belonging to enterobacteriaceae were also common causative organisms in urinary tract infection. In pneumonia, Gram-negative bacilli were more frequently identified as causative pathogens than Gram-positive bacteria. Among Gram-negative bacteria, 57.7\% were non-fermenting, including Pseudomonas aeruginosa, Acinetobacter baumannii, and Stenotrophomonas maltophilia. CMV pneumonia $(\mathrm{n}=$ $1,3.4 \%)$, active pulmonary tuberculosis $(\mathrm{n}=2,6.8 \%)$, and invasive pulmonary aspergillosis $(n=2,6.8 \%)$ were reported in a small number of patients during follow-up. There was no case recorded of $P$. jirovecii pneumonia during follow-up. Coagulase-negative staphylococci (CoNS) were the most commonly isolated organisms in CLABSI ( $\mathrm{n}=13,50 \%$ of total identified CLABSI).

\section{DISCUSSION}

LT recipients are especially vulnerable to infections due to the technical complexity of the surgical procedure and complications related to abdominal surgery and manipulation of the hepatobiliary system [1]. In this study, 89 of 185 patients had infectious complications during the follow-up (48.1\%). This rate is comparable with that found in previous reports (37.3\% to $76.9 \%$ ) $[5,9,10]$. In this study we investigated risk factors for infectious complications during the perioperative period. By analyzing demographic, clinical, and laboratory data, we found that deceased-donor transplantation, high MELD scores, and acute rejection were significantly associated with infectious complications.

In previous studies, a direct association between mortality and severity of preoperative clinical condition in- cluding infection and MELD score, has been observed $[9,11]$. The Italian Association for the Study of the Liver (AISF) has reported risk factors of post-LT infections, including MELD score > 30, level and type of immunosuppression, and primary non-function [12]. However, they did not mention that the risk of infection was increased when organs were derived from deceased-donors compared to when organs were obtained from living-donors [12]. Saner et al. [13] have reported that higher risks of pulmonary and blood stream infections in recipients of living donor organs might be due to smaller liver volumes and longer warm ischemia times. Their study investigated blood-stream infection (BSI) and pneumonia after LT surgery [13] without including other types of infectious complications. On the other hand, another study reported a higher prevalence of bacterial infection in deceased-donor liver transplantation (DDLT) than that in living-donor liver transplantation (LDLT) [14]. The present study was conducted on a larger number of patients with a longer duration of follow-up for each patient than the previous study [14].

In a recently published Korean study, Kim et al. [15] investigated the epidemiology and clinical features of post-transplant BSI in LT recipients. In a 1-year period following LT, 112 episodes of BSI occurred in 64 of the 222 patients (28.8\%) [15]. MELD score was shown as a risk factor of BSI in univariate analysis [15]. On the other hand, the type of donor did not show a significant difference between patients with BSI and patients without BSI [15]. In multivariate analysis, they reported biliary complications and longer ICU stay were significant independent risk factors of post-transplant BSI [15]. The distribution of identified bacteria was relatively similar to our study [15]. On the other hand, our study included not only BSI but also other types of infections and so in this regard our study provides significant new information.

The higher infection rate in the DDLT group in the present study could be due to several reasons. First, most of the patients who received DDLT had high MELD scores with poor systemic condition. Second, screening of transplant donors for infection is limited by available technology and the short period during which organs from deceased-donors can be used [16]. Patients who need liver transplantation have long wait times due to the lack of donors, especially in Korea [17]. Because of this, the priority for deceased-donor LT is given to pa- 
tients with very poor liver function and prognosis [17].

Since the Korea organ-distribution law was passed in 2000, the Child-Pugh score of patients in the waiting list has been used to distribute organs. This was before the liver donor law which passed recently. Since June 1, 2016, the MELD score system instead of Child-Pugh score has been used, based on various studies [10]. The results of the present study provide new data to support the clinical importance of using MELD score instead of Child-Pugh to obtain better prognosis after surgery without infection. This study also showed that the risk of infection was increased in patients with acute rejection. Some studies have shown that the level of immunosuppression is a risk factor of infection after LT $[2,6]$. Possibly, patients with acute rejection are more vulnerable to infection because of the increased amount of immunosuppressive agents used.

Bacterial infections were the major causes of infectious complications, followed by fungal and viral infections. Among the causative bacterial species identified, Enterococcus species were leading pathogens, followed by E. coli and Klebsiella species. This is consistent with results of recent studies $[2,5,6]$. The most common infections in this study were intra-abdominal including infections of biliary tract. This was followed by urinary tract infections, pneumonia, and CLABSI. Although bundle approaches have been applied in our institution to prevent VAP, CLABSI, and catheter associated-urinary tract infections, these infections were found to be common in LT patients.

Gram-negative bacteria were found to be more common compared to Gram-positive bacteria among pathogens identified in pneumonia in this study. This is similar to results from a previous report about hospital-acquired pneumonia [2,18]. Non-fermenting gram-negative bacilli found in hospital environments, are reported to be responsible for hospital acquired infections [19]. Interestingly, S. maltophilia and A. baumannii were found to be important causative agents of pneumonia in our institution. The distribution of causative organisms of pneumonia in this study may be meaningful when selecting appropriate antibiotics.

CoNS accounted for half of the causative organisms in CLABSI. Therefore, better CLABSI prevention approaches are needed to achieve better management for LT patients. Kaido et al. [11] have reported that central venous catheterization of LT patients is significantly lowered through rapid central venous catheter removal and rapid intestinal dietary intake. To prevent CLABSI and catheter-associated urinary tract infection, it might be important to check appropriate implementation of bundle approach in all patients and consider rapid removal as soon as possible.

In this study, patients undergoing surgery after 2010 were included. In a previous study using accumulated data from the last several years, a shift toward increased Gram-negative bacterial infections and emergence of multi-drug resistant bacterial pathogens was documented [4]. Enterococcus species were found to be major pathogens in LT patients in the present study. From this point of view, clinicians should pay close attention to Enterococcus, including vancomycin-resistant enterococci (VRE), in LT patients because the clinical importance of infection or colonization during the perioperative period due to VRE has been reported [20].

This study has several limitations. First, it was a retrospective study. Second, we included three children aged 10 years or older in this study. Third, as a single center study, the number of patients participating in the study was not as high as we would have liked. Fourth, this study did not review surgical techniques or intraoperative transfusions. Despite these limitations, this study was meaningful in that it was conducted with a considerable number of LT patients in Korea since 2010. The number of LTs performed in our institution in 5 years was more than that in other institutions of Korea as single centers.

In conclusion, high MELD score, deceased-donor type, and presence of acute rejection are associated with infectious-related morbidity of LT patients in this study. Since infection after liver transplantation is closely associated with postoperative prognosis, MELD score should be considered as an indicator when determining LT (DDLT or LDLT) recipients, before liver function deteriorates further.

\section{KEY MESSAGE}

1. High preoperative Model for End-Stage Liver Disease (MELD) score, deceased-donor type and acute rejection were risk factors associated with infection. 
2. It is important to determine the appropriate time of operation before the recipient has high MELD score.

\section{Conflict of interest}

No potential conflict of interest relevant to this article was reported.

\section{REFERENCES}

1. Freire MP, Pierrotti LC, Oshiro IC, et al. Carbapenem-resistant Acinetobacter baumannii acquired before liver transplantation: impact on recipient outcomes. Liver Transpl 2016;22:615-626.

2. Kim SI. Bacterial infection after liver transplantation. World J Gastroenterol 2014;20:6211-6220.

3. Rostved AA, Lundgren JD, Hillingso J, Peters L, Mocroft A, Rasmussen A. MELD score measured day 10 after orthotopic liver transplantation predicts death and re-transplantation within the first year. Scand J Gastroenterol 2016;51:1360-1366.

4. Karapanagiotou A, Kydona C, Papadopoulos S, et al. Infections after orthotopic liver transplantation in the intensive care unit. Transplant Proc 2012;44:2748-2750.

5. Kim YJ, Kim SI, Wie SH, et al. Infectious complications in living-donor liver transplant recipients: a 9-year single-center experience. Transpl Infect Dis 2008;10:316-324.

6. Hamandi B, Husain S, Grootendorst P, Papadimitropoulos EA. Clinical and microbiological epidemiology of early and late infectious complications among solid-organ transplant recipients requiring hospitalization. Transpl Int 2016;29:1029-1038.

7. Ljungman P, Griffiths P, Paya C. Definitions of cytomegalovirus infection and disease in transplant recipients. Clin Infect Dis 2002;34:1094-1097.

8. De Pauw B, Walsh TJ, Donnelly JP, et al. Revised definitions of invasive fungal disease from the European Organization for Research and Treatment of Cancer/Invasive Fungal Infections Cooperative Group and the National Institute of Allergy and Infectious Diseases Mycoses Study Group (EORTC/MSG) Consensus Group. Clin Infect Dis 2008;46:1813-1821.
9. Avkan-Oguz V, Ozkardesler S, Unek T, et al. Risk factors for early bacterial infections in liver transplantation. Transplant Proc 2013;45:993-997.

10. Vera A, Contreras F, Guevara F. Incidence and risk factors for infections after liver transplant: single-center experience at the University Hospital Fundacion Santa Fe de Bogota, Colombia. Transpl Infect Dis 2011;13:608-615.

11. Kaido T, Egawa H, Tsuji H, Ashihara E, Maekawa T, Uemoto S. In-hospital mortality in adult recipients of living donor liver transplantation: experience of 576 consecutive cases at a single center. Liver Transpl 2009;15:1420-1425.

12. Fagiuoli S, Colli A, Bruno R, et al. Management of infections pre- and post-liver transplantation: report of an AISF consensus conference. J Hepatol 2014;60:1075-1089.

13. Saner FH, Olde Damink SW, Pavlakovic G, et al. Pulmonary and blood stream infections in adult living donor and cadaveric liver transplant patients. Transplantation 2008;85:1564-1568.

14. Varghese J, Gomathy N, Rajashekhar P, et al. Perioperative bacterial infections in deceased donor and living donor liver transplant recipients. J Clin Exp Hepatol 2012;2:35-

15. Kim HK, Park YK, Wang HJ, et al. Epidemiology and clinical features of post-transplant bloodstream infection: an analysis of 222 consecutive liver transplant recipients. Infect Chemother 2013;45:315-324.

16. Fishman JA. Infection in solid-organ transplant recipients. N Engl J Med 2007;357:2601-2614.

17. Kim MS. Modification of emergency status in deceased donor liver allocation: evidence for Korean Model of End-stage Liver Disease (MELD) system. J Korean Soc Transplant 2016;30:51-58.

18. Zhao T, Liu Y, Cao B, et al. Prospective multicenter study of pathogen distributions in early-onset and late-onset hospital-acquired pneumonia in china. Antimicrob Agents Chemother 2013;57:6404-6405.

19. Sharan H, Katare N, Pandey A, Bhatambare GS, Bajpai T. Emergence of hospital acquired carbapenem resistant non-fermenters in teaching institute. J Clin Diagn Res 2016;10:DC20-DC23.

20. Kim YJ, Kim SI, Choi JY, Yoon SK, You YK, Kim DG. Clinical significance of methicillin-resistant Staphylococcus aureus and vancomycin-resistant enterococci colonization in liver transplant recipients. Korean J Intern Med 2015;30:694-704. 